\title{
La sexualidad de las personas con lesión medular: aspectos psicológicos y sociales. Una revisión actualizada
}

\author{
Sexuality of spinal cord injured persons: \\ psychological and social aspects. An updated \\ review
}

\author{
LARA GARCÍA CARENAS* \\ M. a ÁNGELES ALCEDO RODRÍGUEZ* \\ ANTONIO-LEÓN AGUADO DÍAZ*
}

\section{RESUMEN}

Este artículo tiene como objetivo presentar una revisión de la investigación sobre los aspectos psicológicos y sociales relacionados con la sexualidad de las personas con lesión medular (LM). La importancia que el impacto de la lesión tiene sobre la persona y su entorno, en general, y sobre la esfera de la sexualidad, en particular, la convierte en un área de gran interés científico y social en la actualidad. Esto se explica por el crecimiento constante en la prevalencia y la radicalidad de las alteraciones biopsicosociales que conlleva la LM. Así, en su vida sexual la persona ha de afrontar cambios tanto en lo fisico como en lo emocional y relacional. A tal efecto, lo que pretendemos es revisar la bibliografia disponible, intentando abordar y delimitar sucesivamente i) el tipo de publicaciones en las que se trata el tema, ii) las áreas temáticas de estudio iii) la metodología de las investigaciones, para llegar, por último, iv) a las conclusiones de nuestra revisión.

\section{PALABRAS CLAVE}

Lesión medular, Sexualidad, Discapacidad fisica, Asesoramiento psicológico, Problemas metodológicos, Revisión.

\footnotetext{
* Departamento de Psicología. Universidad de Oviedo.
} 
La sexualidad de las personas con lesión medular: aspectos psicológicos y sociales. Una revisión actualizada

\section{ABSTRACT}

This article presents a research review on the psychological and social aspects related with the sexuality of persons with spinal cord injury. The relevance that the impact of injury has on people and its environment, in general, and on sexuality sphere in particular, becomes an area of great scientific and social interest. This is explained by the constant growth in the prevalence and in the radical bio-psycho-social changes that the spinal cord injury involves. So, people must cope in their sexual life with changes as much in the physical as in the emotional areas as well as in social relationships. With this purpose, the available bibliography is revised trying to focus in i) the size of publications where the topic appears in, ii) the thematic areas object of study, iii) the publications methodology, to reach finally iv) the conclusions of our review.

\section{KEY WORDS}

Spinal cord injury, Sexuality, Physical disability, Psychological guidance, Methodological problems, Review. 


\section{INTRODUCCIÓN}

En el año 1972 fueron por primera vez abordados los derechos sexuales de las personas con discapacidad en el Congreso Mundial de Rehabilitación (Chigier, 1981). Casi tres décadas después, la declaración aprobada por la Asamblea General de la Asociación Mundial de Sexología en el $14^{\circ}$ Congreso Mundial de Sexología, celebrado en el año 1999, recoge los derechos sexuales de las personas con discapacidad como derechos universales basados en la libertad, dignidad e igualdad inherentes a todos los seres humanos. (Asociación Mundial de Sexología, 1999). Se postula que la persona con discapacidad es sujeto de derechos, fundamentalmente el derecho natural e inherente a toda persona de vivir, expresar y disfrutar de su sexualidad de la manera y forma que desee. Se considera la sexualidad como una dimensión global que mediatiza la totalidad del ser humano con independencia de su dotación intelectual o de sus habilidades motoras. Sin embargo, y pese a la modificación de las normas de conducta sexual que se han producido en los últimos treinta años, modificación que se ha traducido en una mayor flexibilidad en el enfoque de cuestiones relacionadas con la libertad sexual y el derecho a expresarnos sexualmente, la afirmación del derecho a la sexualidad de las personas con discapacidad plantea más de un espinoso dilema, tanto en la familia como en el grupo social en su conjunto. Ha sido objeto de tabú durante largos años y ha estado sometida a falsos mitos y falacias, pese a constituir un aspecto relevante y de vital importancia en el proceso de adaptación y ajuste a la LM. El abordaje de la sexualidad debe ser considerado parte esencial del "proceso de integración social" de las personas con LM (PcLM). Este colectivo demanda y reclama frecuentemente asesoramiento sobre el tema, pese a que sólo un pequeño porcentaje recibe información al respecto (Anderson, Mulcahey y Vogel, 1997; Charlifue, Gerhart, Menter, Whiteneck y Manley, 1992; Fitting et al., 1978; Richards, Tepper, Whipple y Komisaruk, 1997; Sawyer, Allen, Reisin, 1983; Tepper, Whipple, Richards y Komisaruk, 2001; Zwerner, 1982).

Nuestro interés con esta revisión bibliográfica radica en conocer cómo se encuentra la literatura científica en el ámbito de la sexualidad en PcLM, en concreto, en el apartado relativo a sus aspectos psicológicos y sociales, y en comprobar hasta que punto la investigación en este campo se está realizando con el rigor metodológico preciso. Para conocer la literatura existente sobre dicho tema, nuestro primer paso consistió en hacer una búsqueda bibliográfica en las principales bases documentales, Psycinfo, Psicodoc, Medline y Scopus. Las palabras clave que hemos utilizado como descriptores han sido: "Sexuality", "Physical Disability" y "Spinal Cord Injury". Obtuvimos un total de 161 referencias bibliográficas. De todas las referencias encontradas cabe destacar que muchas de ellas guardan relación con aspectos médicos y neurológicos, más que psicológicos propiamente dichos, que son los que realmente nos interesan, lo que nos ha llevado a desestimar algunas de estas referencias. Además, y debido a la escasez de artículos encontrados, nos hemos tenido que remontar a publicaciones de hace 30 años para poder observar la evolución que la investigación sobre este tema ha sufrido en las tres últimas décadas. Finalmente, hemos seleccionado un total de 78 artículos que aluden de forma explícita a la sexualidad de las PcLM.

En consecuencia, los objetivos específicos de esta revisión se han centrado en i) el análisis del volumen de publicaciones que incluyen los descriptores ante- 
riormente mencionados en las fuentes documentales al uso, para conocer así el tipo de publicaciones en las que se aborda el tema, ii) el estudio de las áreas temáticas que han despertado un mayor interés y, iii) el examen de la metodología utilizada en las investigaciones.

\section{TIPO DE PUBLICACIONES CENTRADAS EN LA SEXUALIDAD DE LAS PERSONAS CON LM}

El primero de los objetivos que nos planteamos fue acercarnos al tipo y clase de publicaciones que abordan la sexualidad en PcLM. Los 78 artículos seleccionados, de las más de 160 publicaciones revisadas, pueden agruparse en varias categorías, en concreto, artículos en publicaciones periódicas, manuales $y$ páginas web.

Los artículos aparecen diseminados en un amplio abanico de publicaciones periódicas y de revistas. A lo largo de la revisión bibliográfica, hemos encontrado revistas, no especializadas en el ámbito de la discapacidad, que en alguno de sus capitulos tratan el tema de la sexualidad en las PcLM tanto desde el ámbito de la medicina (Journal of Psychosocial Nursing \& Mental Health Services, Neurology y Urology), como desde el de la psicologia propiamente dicho (Archives of Sexual Behavior, British Journal of Clinical Psychology, Journal Sex \& Marital Therapy, Psychology: A Journal of Human Behavior y Terapia Sexual y de Pareja). Por otro lado, aparecen revistas especializadas en discapacidad que dedican articulos de sus publicaciones al tema de la sexualidad y la LM (Scandinavian Journal of Rehabilitation Medicine Supplement, Journal of Applied Rehabilitation Counseling, Archives of Physical Medicine and Rehabilitation, Reproductive Issues for Persons with Physical Disabilities, Sexuality and Disability, Boletín del Real
Patronato y Rehabilitation). Y, por último, surge otro grupo de publicaciones específicas en LM (Paraplejia, SCI Nursing: A publication of the American Association of Spinal Cord Injury Nurses y Spinal Cord), donde también se aborda el tema de la sexualidad.

Los manuales encontrados referentes a nuestro tema de estudio podemos clasificarlos en varios grupos. En primer lugar, los manuales específicos sobre la sexualidad de las PcLM (Béjar y Béjar, 1989; Curcoll y Vidal, 1992; De Pinto y Sánchez, 2000; Ducharme y Gill, 1997). A continuación, los capítulos específicos sobre sexualidad en manuales generales sobre LM (Giner, 2001). En tercer lugar, los capítulos específicos sobre sexualidad en manuales generales sobre discapacidad (Blanchard, 1988; Cardenal, 1993; Conine, 1988; Edmonson, 1988; Zaretsky, 1981). En cuarto lugar, los capítulos especificos sobre sexualidad en manuales generales sobre sexualidad y discapacidad (Chigier, 1981; Sipski, 1993; Tepper, 1997). Por último, referencias a la sexualidad de las PcLM en capítulos, o partes del capítulo, en alguno de los casos anteriores (Aguado, 1995; Aguado y Alcedo, 1995, 1999; Brucker, 1983; Burish y Bradley, 1983; Freed, 1993).

De igual forma, encontramos diferentes páginas web que abordan el tema de la sexualidad y la LM. Así, las páginas oficiales de los dos hospitales más relevantes de nuestro país en el tema de la rehabilitación de la LM, el "Hospital Nacional de Parapléjicos" de Toledo y la "Fundación Privada Instituto de Neurorrehabilitación Guttmann" de Barcelona, hacen referencia específica al tema. Otras páginas interesantes en el ámbito internacional son la "Fundación Christopher Revé", el "Proyecto Miami para la cura de la parálisis" y el "Proyecto Stand Up and Walk". 
En sintesis, y ante la variedad de fuentes documentales en las que hemos obtenido información sobre el tema objeto de estudio, cabe destacar que la revisión ha mostrado escasos trabajos de investigación en castellano, frente al dominio de estudios americanos y británicos. Sin embargo, sí que contamos con un buen volumen de monografias y de capítulos de libros en castellano acerca de la sexualidad de las PcLM. Ahora bien, el acercamiento al tema se realiza frecuentemente desde planteamientos de naturaleza teórica o bien desde estudios de corte descriptivo-correlacional, frente a la escasez y prácticamente ausencia de investigación aplicada y estudios empiricos.

\section{ÁREAS TEMÁTICAS DE INTERÉS}

Otro de nuestros objetivos se ha centrado en el análisis de las áreas temáticas de estudio que han interesado a los investigadores. En general, estas publicaciones se centran en aspectos variados que incluyen desde variables predictoras relacionadas con la actividad sexual hasta técnicas conductuales para el tratamiento de la disfunción eréctil. Ahora bien, el género establece diferencias importantes en el tipo de áreas temáticas abordadas. Por ello, se han diferenciado los estudios relacionados con los varones lesionados medulares, los cuales han sido elemento prioritario de investigación a lo largo de la historia, tanto desde el campo de la medicina como desde la psicologia, de aquellos otros centrados en mujeres con LM, que hacen referencia principalmente a temas de fertilidad y reproducción. Asimismo, se ha optado por presentar de forma diferenciada los trabajos relativos a los profesionales de la salud, dada la relevancia de su rol en la rehabilitación física y psicológica del colectivo de PcLM (cuadro 1).
Así, comenzando con el primero de los grupos, los varones con $L M$, en general estas publicaciones hacen hincapié en la incidencia de la lesión en el nivel de actividad y satisfacción sexual. Se constata el descenso y disminución significativa de contactos sexuales después de la lesión (Phelps, Brown, Chen, Dunn, Lloyd, Stefanick, y Davidson , 1983), y se señalan como factores determinantes para continuar manteniendo relaciones sexuales el nivel y la severidad de la lesión (Sjogren y Egberg, 1983), así como la existencia o ausencia de pareja antes de padecer la lesión (Phelps, Albo, Dunn y Joseph, 2001), la adaptación a las limitaciones sexuales, la satisfacción con la pareja, el autoconcepto sexual y la calidad y cantidad de sus relaciones sexuales (Berkam, Weissman y Frielich, 1978), entre otros.

En cuanto a los tratamientos para la disfunción eréctil, se hace referencia a estrategias conductuales basadas en la activación del músculo perineal capaces de provocar erecciones en varones con LM (Courtois, Mathieu, Charvier, Leduc y Bélanger, 2001), y se reconoce la importancia del asesoramiento sexual, aspecto que se considera fundamental en la rehabilitación del varón con LM, con el objetivo de reeducar y reorientar la sexualidad hacia conductas y vivencias satisfactorias y placenteras (Rohme, 1979). Asimismo, se señala el decisivo rol que puede desempeñar la pareja en el tratamiento de los problemas de disfunción eréctil (Yoshida, 1994).

En cuanto al segundo de los subgrupos, las mujeres con $L M$, se enfatiza la importancia que la sexualidad desempeña en sus vidas, indicando que una vez pasado el shock inicial la sexualidad vuelve a ocupar un lugar prioritario (Leibowitz, 2003; Whipple, Richards, Tepper y Komisaruk, 1996). Interesa, asimismo, el tipo de contacto sexual, destacando la 
La sexualidad de las personas con lesión medular: aspectos psicológicos y sociales. Una revisión actualizada

\section{Cuadro 1. Principales áreas temáticas abordadas en las investigaciones sobre} sexualidad y LM

\begin{tabular}{|c|c|c|}
\hline GRIJPOS & $\Lambda \mathrm{RE} / \mathrm{S}$ TEM $\Lambda \mathrm{TIC} \Lambda \mathrm{S}$ & NUTORES \\
\hline VARONES & $\begin{array}{l}\text { - Nivel de actividad y satisfacclón } \\
\text { sexual } \\
\text { - Tratamlentos disfunclón erèctil. }\end{array}$ & $\begin{array}{l}\text { Berkam et al., 1978; Phelps, 1983; } \\
\text { Phelps et al., 2001; Sjogren y Egberg. } \\
1983 \text {; Phclps ct al., 2001. } \\
\text { Courtols et. al., 2001; Rohme, 1979; } \\
\text { Yoshida, 1994. }\end{array}$ \\
\hline MUJERES & $\begin{array}{l}\text { - Tlpo de contacto sexual. } \\
\text { - Cambios corporales y } \\
\text { perceptlvos. } \\
\text { - Importancia de lit scxualidad y } \\
\text { de la educaclón sexual. } \\
\text { - Problemas incontinencla, } \\
\text { espasticidad, madurez. }\end{array}$ & $\begin{array}{l}\text { Black et al., 1998; Sipski y Nlexander, } \\
1993 . \\
\text { Ferrciro-Vclasco ct al., 2004; Fitting ct } \\
\text { al., 1978; Harrison et. al., 1995; Kettl, } \\
\text { 1991; Lysberg y Severinsson, } 2003 . \\
\text { Andcrson ct al., 1997; Charliluc ct al., } \\
\text { 1992; Fitting et. al., 1978; Richards et. al, } \\
\text { 1997; Sawyer et al.. 1983; Tepper et al., } \\
\text { 2001; Zwcrncr, 1982. } \\
\text { Penthland et. al., 2002 ; Westgren et al., } \\
\text { 1997. }\end{array}$ \\
\hline $\begin{array}{l}\text { PROFESIONA- } \\
\text { I.ES }\end{array}$ & $\begin{array}{l}\text { Conocimiento y experlencla. } \\
\text { - Creenclas y experienclas } \\
\text { personales } \\
\text { - Ilabilidades socialcs y relacioncs } \\
\text { empáticas. } \\
\text { - Sistcmas y matcriales dc } \\
\text { trabajo. }\end{array}$ & $\begin{array}{l}\text { Conine et. al., 1979; Held et. al., 1975; } \\
\text { Lemon, 1993; McBride y Kines, 2000; } \\
\text { Novak y Mitchell, 1988; Sawycr y Allcn, } \\
1983 . \\
\text { Alexander, 1991: Girts, } 1990 . \\
\text { Anderson y Cole, 1975; Cole y Stevens, } \\
\text { 1975: Evans et al., 1976; Kendall et al., } \\
2003 \text {. } \\
\text { Rooth et. al., 2003; Herson et al., 1999; } \\
\text { Ward-McKinlay et al., } 1983 .\end{array}$ \\
\hline
\end{tabular}

importancia que adquieren para las mujeres las caricias, los besos y los juegos sexuales, más allá del coito propiamente dicho (Sipski y Alexander, 1993). También se hace mención a los cambios significativos entre el antes y el después de la LM en la frecuencia de las relacio- nes sexuales y en la capacidad para sentir y percibir los orgasmos. La gran mayoría de las mujeres con LM continúan, aunque sea de forma más esporádica, manteniendo relaciones sexuales una vez sufrida la LM, manifestando en algunos casos que su vida sexual les resulta 
menos espontánea y satisfactoria (Black, Sipski y Strauss, 1998). Los cambios en la percepción de sus cuerpos también resulta un tema abordado, señalándose que ello no es obstáculo para seguir manteniendo una vida sexual activa (Ferreiro-Velasco, Barca-Buyo, Salvador de la Barrera, Montoto-Marqués, Miguéns y Rodríguez-Sotillo, 2004; Fitting, Salisbury, Davies y Mayclin, 1978; Harrison, Glass, Owens y Soni, 1995; Kettl, 1991; Lysberg y Severinsson, 2003). De igual forma, se abordan los problemas relacionados con la incontinencia urinaria, la espasticidad y las limitaciones de movilidad, aspectos que pueden interferir en las actividades sexuales de las mujeres con LM (Westgren, Hultling, Levi Seiger y Westgren, 1997), así como el impacto del proceso de envejecimiento en esta área de la sexualidad (Pentland, Walker, Minnes, Tremblay, Brouwer y Gould, 2002).

La importancia y la implicación de la educación sexual y del asesoramiento en los cambios que sufren las mujeres con LM también constituyen temáticas frecuentemente abordadas. Se reclama con insistencia este tipo de asesoramiento, ya que no resulta habitual pese a ser ampliamente demandado. Se aboga por la necesidad de una guía de educación y asesoramiento sexual en el proceso de rehabilitación y de una adecuada transmisión de la información (Anderson, Mulcahey y Vogel, 1997; Charlifue, Gerhart, Menter, Whiteneck y Manley, 1992; Fitting et al., 1978; Richards, Tepper, Whipple y Komisaruk, 1997; Sawyer, Allen, Reisin, 1983; Tepper, Whipple, Richards y Komisaruk, 2001; Zwerner, 1982).

Por lo que se refiere al papel que juegan los profesionales en el campo de la rehabilitación sexual de las PcLM, otro de los tópicos temáticos encontrados en esta revisión, cabe destacar que son muchos los profesionales que admiten su desconocimiento sobre el tema de la sexualidad en la LM y la falta de información al respecto (Held, Cole, Anderson y Chilgren, 1975; Conine et al., 1979; Lemon, 1993; McBride y Rines, 2000; Sawyer y Allen, 1983). Es más, algún estudio pone de manifiesto que algunos de estos profesionales no consideran el asesoramiento a las PcLM como parte fundamental o importante en su trabajo (Novak y Mitchell, 1988).

De igual forma, se alerta de la interferencia negativa que las propias creencias o pensamientos de los profesionales acerca de la sexualidad pueden ocasionar en el asesoramiento y en la información que transmitan a sus pacientes (Girts, 1990; Alexander, 1991), y se señalan las habilidades terapéuticas que posibilitan un mejor asesoramiento, así como el establecimiento de relaciones más empáticas (Anderson y Cole, 1975; Cole y Stevens, 1975; Evans, Halar, DeFreece y Larsen, 1976; Held, Cole, Anderson y Chilgren, 1975; Kendall, Booth. Fronek, Miller y Geraghty, 2003). También se informa acerca de las técnicas y materiales de trabajo que resultan más eficaces, tales como películas de educación sexual, libros y manuales, role-playing, conversaciones y discusiones abiertas con los pacientes, etc., que permiten que la persona con LM adopte actitudes mucho más positivas, aprenda a distinguir entre los aspectos físicos y los emocionales y demande más apoyo terapéutico sobre temas relacionados con la sexualidad (Herson, Hart, Gordon y Rintala, 1999).

En la última década las publicaciones centradas en esta temática relativa al entrenamiento y la formación de los profesionales se han incrementado considerablemente, lo que ha tenido un impacto positivo en la sexualidad de estas personas (Booth, Kendall, Fronek, Miller y 
Geraghty, 2003; Ward-McKinlay, Botvinn-Madorsky y Ward-McKinlay, 1983). Además, ya son varios los autores que han propuesto la elaboración de guías 0 manuales de orientación sexual para pacientes con LM (Closson, Toerge, Ragnarsson, Parsons y Lammertse, 1991; Ferolla y Lourenco, 1996 y Garcés y Civera, 1997).

A continuación presentamos el último de los objetivos propuestos en esta revisión, el análisis de la metodologia utilizada en las investigaciones sobre LM y sexualidad.

\section{METODOLOGÍA UTILIZADA}

Las publicaciones encontradas las hemos clasificado en dos grandes grupos (cuadro 2). En primer lugar, todos aquellos estudios de naturaleza teórica en los que no aparecen datos empíricos, concretamente, 26 de los 78 estudios revisados, es decir, un 33,33\% del total. Estos trabajos los hemos subdividido a su vez en revisiones bibliográficas (12 revisiones, un 15,38\%), reflexiones por parte de los autores (11 reflexiones, un 14,10\%) y guias de orientación sexual (3 estudios, un $3,85 \%$ ).

En segundo lugar, se presentan aquellas publicaciones que aportan algún tipo de dato empirico, en concreto, 52 estudios, un $66,66 \%$ del total. Este grupo lo hemos subdividido en estudios de corte descriptivo-correlacional (48 estudios, un $61,54 \%$ ) y en investigaciones experimentales (4 estudios, un 5,12\%).

\section{ESTUDIOS DE NATURALEZA TEÓRICA}

Del total de estudios revisados sobre el tema (26 artículos), 12 de ellos son revisiones bibliográficas, 11 reflexiones de los autores, y los 3 artículos restantes corresponden a guias de orientación sexual dirigidas a PcLM y a sus cuidadores.

Por lo que atañe a las revisiones teóricas, en concreto 9 de las 12 totales, se centran en discutir cómo se encuentra la literatura cientifica en torno a la sexualidad de las PcLM, sin hacer distinción de género. Así, autores como Miller (1975), Teal y Athelstan (1975) y Willmuth (1987), subrayan el desconocimiento en torno a la sexualidad de las PcLM, y critican los resultados difusos de las investigaciones y los problemas metodológicos. Otros, como Evans et al. (1976) y Higgins (1979) demandan la utilización de técnicas psicológicas en los procesos de rehabilitación para así constatar los efectos derivados de la intervención. Por su parte, Drench (1992) y Milligan y Neufeldt (2001) hablan de las actitudes y de las emociones considerando que los problemas de ajuste y de rehabilitación que sufre este colectivo se reflejan con

Cuadro 2. Distribución de las publicaciones en función de la metodología utilizada

\begin{tabular}{|l|l|l|}
\hline Metodologia & № & $\%$ \\
\hline Teórica: & 26 & 33,33 \\
- Revisiones & 12 & 15,38 \\
- Reflexiones & 11 & 14,10 \\
- Guías & 3 & 3,85 \\
\hline Empírica: & 52 & 66,66 \\
- Descriptivo-correlacionales & 48 & 61,54 \\
- Experimentales & 4 & 5,12 \\
\hline Total & $\mathbf{7 8}$ & $\mathbf{1 0 0}$ \\
\hline
\end{tabular}


claridad en sus relaciones sociales y personales. Por último, Sipski (1998) y Singer (2003) analizan en sus artículos las alteraciones y los cambios que se producen en el funcionamiento sexual cuando a una persona le sobreviene una LM.

Por otra parte, entre aquellos autores que tienen en cuenta la perspectiva de género, merece la pena destacar el estudio de Derry et al. (2002), revisión que aborda la eficacia de la "viagra" (Sildenafil) en el tratamiento de la disfunción eréctil en los varones con LM. Por su parte, Berard (1989) y Sipski (1993) se centran en la evaluación de la disfunción sexual de mujeres con LM, y denuncian las limitaciones metodológicas de este tipo de estudios. Consideran que los cuestionarios que se pasan a las mujeres para evaluar los efectos que la lesión causa en su vida sexual deben ir acompañados de un detallado y riguroso examen neurológico. Además, el proceso de evaluación requerirá exámenes psicológicos más profundos que los meros autoinformes que los sujetos cumplimentan sobre sí mismos.

En lo referente al apartado de las reflexiones por parte de los autores, en las 11 publicaciones analizadas (Alexander, 1991; Anderson y Cole, 1975; Chicano, 1989; Garcés y Civera, 1997; Girts, 1990; Herson et al., 1999; Pervin-Dixon, 1988; Sawyer y Allen, 1983; Spica, 1989 y Ward-McKinlay et al., 1983), los autores coinciden en afirmar que aunque los temas relativos a la sexualidad de las PcLM han recibido creciente importancia en las últimas décadas, también es cierto que los aspectos sexuales, tanto desde la perspectiva médica como desde la psicológica, tienden a resultar embarazosos e incómodos para los profesionales y, por ello, son obviados en muchas ocasiones. Los autores ratifican en sus trabajos que un tratamiento multidisciplinar e integrador y un asesoramiento adecuado a todos los niveles son elementos suficientes para que el proceso de rehabilitación sea exitoso y eficaz.

Por último, hemos recogido tres artículos que tienen por objetivo la elaboración de unas guías de orientación y asesoramiento sexual tanto para pacientes con LM como para sus cuidadores. El propósito de las mismas es ayudar a estas personas a expresar su sexualidad de la forma más adecuada. Dichas guías son elaboradas por el personal sanitariorehabilitador (Closson et al., 1991; Ferolla y Lourenco, 1996) o por las propias PcLM mediante formatos de preguntarespuesta (Ducharme y Gill, 1997).

En sintesis, la mayor parte de estos trabajos de naturaleza teórica concluyen abogando por la necesidad de continuar investigando en este campo para así contar con datos más sólidos sobre los que asentar el proceso de rehabilitación integral de las PcLM. Se constata que el área de la sexualidad no es un tema suficientemente abordado y estudiado desde planteamientos científicos, tampoco exento de problemas metodológicos, y obviado con demasiada frecuencia desde el ámbito de la rehabilitación.

\section{ESTUDIOS EMPÍRICOS}

Bajo este epígrafe de estudios empíricos (52 artículos) vamos a presentar aquellas investigaciones sobre sexualidad en PcLM que incluyen en sus diseños una metodología descriptivo-correlacional (48 artículos) o experimental (4 artículos), y que contemplan, por tanto, la recogida de datos y la utilización de instrumentos estandarizados de evaluación.

El primero de los grupos, los descriptivo-correlaciones, engloba el abanico más amplio de estudios sobre el tema. Las 
propias PcLM, las parejas que conviven con ellas y el personal sanitario son los principales colectivos estudiados. Así, encontramos estudios que abordan por separado, varones, por un lado, (Phelps et al., 2001; Phelps et al, 1983 y Sjogren y Egberg, 1983), y mujeres, por otro, (Anderson et al., 1997; Black et al.,1998; Charlifue et al., 1992; Ferreiro-Velasco et al., 2004; Harrison et al., 1995; Kettl, 1991;Leibowitz, 2003; Lysberg y Severinsson, 2003; Pentland et al., 2002; Richards et al., 1997; Sipski y Alexander, 1993; Tepper et al., 2001; Westgren et al., 1997; Whipple et al.,1996), temas relativos a los factores asociados con la satisfacción sexual, las experiencias y tipos de relaciones vividas por estas personas, las necesidades y los problemas de ajuste sexual y los cambios aparecidos en esta esfera tras la lesión. Otros, por el contrario, comparan las diferencias en función del género en aspectos variados, p.e., experiencias, impacto emocional, autoestima, servicios de orientación, etc., (Benony et al., 2002; Donohue y Gebhard, 1995; Fisher et al., 2002; Guldin, 2000; Ide y Ogata, 1995; Lesky, 2001; Low y Zubir, 2000; McAlonan, 1996; Reitz, Tobe, Knapp y Schurch, 2004; Taleporos y McCabe, 2001; Tepper, 2002).

En cuanto a las publicaciones que analizan el punto de vista de las parejas que conviven con PcLM, aparecen dos trabajos centrados en las vivencias, sentimientos y cambios que han experimentado sus vidas cuando a sus compañeros sentimentales les sobrevino la lesión (Kreuter, Sullivan y Siosteen, 1994a, 1994b). Por último, aparecen estudios que investigan la problemática de estas personas informada por el personal sanitario y rehabilitador (Booth et al., 2003; Cole y Stevens, 1975; Conine et al., 1979; Lemon, 1993; McBride y Rines, 2000; Novak y Mitchell, 1988; Rohme, 1979).
Una tónica general en este tipo de investigaciones es la presencia de problemas metodológicos, situación esta no exclusiva del ámbito de la sexualidad sino que también afecta a otras áreas de la discapacidad y de la rehabilitación (Aguado, Alcedo y Rueda, 2003). En primer lugar, uno de los errores más frecuentes se deriva de la composición de la muestra. De esta forma, el tamaño muestral de la mayor parte de las investigaciones revisadas resulta poco representativo tanto en número como en variabilidad. Todo ello provoca problemas a la hora de generalizar los resultados. Ejemplos de estas carencias metodológicas son los estudios de Derry et al. (1998), Courtois et al. (2001), Fitting et al. (1978), Kettl (1991) y Yoshida (2004), donde la población no supera los 30 sujetos, así como los trabajos de Fitting (1978), Rohme (1979), Ulmer (1990) y Sipski y Alexander (1993), llevados a cabo en centros hospitalarios.

En segundo lugar, y respecto a los instrumentos de evaluación utilizados, es frecuente la publicación de investigaciones en las que no se mencionan las pruebas que se pasan a los participantes en el estudio y, menos aún, datos acerca de sus criterios de bondad. En determinados trabajos, como los de Kettl (1991), Lysberg y Severinsson (2003), Sjogren y Egberg (1983), Waday Brodwin (1975) y Zwerner (1982), la norma general es omitir los nombres de los cuestionarios o escalas que son utilizadas como instrumentos de evaluación, lo cual hace cuestionable la validez de los mismos. Afortunadamente, hemos encontrado algunas publicaciones que sí aportan el nombre y el tipo de prueba que utilizan en sus estudios (Black et al, 1998, Tepper, 2002, Kendall et al., 2003, Siosteen et al., 1990, Ide y Ogata, 1995, Taleporos y McCabe, 2001, Fisher et al., 2002). 
En tercer lugar, las limitaciones en el diseño de las investigaciones. Un tema pendiente dentro de los estudios descriptivo-correlacionales es la ausencia de grupos de control, pues del total de las investigaciones revisadas con este tipo de diseño sólo en dos de ellas (Benony et al., 2002; Kreuter et al., 1996) los autores refieren la existencia de grupos control con los que comparar los resultados obtenidos en el grupo experimental.

En cuarto y último lugar, queremos destacar la escasez de estudios longitudinales. Así, de los 78 artículos revisados, sólo hemos encontrado un estudio de este tipo, en concreto, el trabajo de Fisher et al. (2002).

Y ya para finalizar este apartado, es preciso hacer mención de las cuatro investigaciones experimentales halladas en esta revisión. Los cuatro estudios centran su objetivo central en comprobar la eficacia de diferentes tratamientos aplicados a PcLM. En primer lugar, el estudio de Courtois et al. (2001) trata de comprobar la eficacia de estrategias conductuales, basadas en la activación del músculo perineal, para optimizar los efectos de estimulación de las glándulas del pene y conseguir erecciones en varones que previamente presentaban disfunciones eréctiles. Sawyer et al. (1983) comprueban la eficacia de un modelo de "microcounseling", es decir, de terapia individual en el tratamiento rehabilitador de la LM. Por su parte, Held et al. (1975) centran sus objetivos en evaluar la eficacia de un programa rehabilitador entre profesionales sanitarios. Por último, Derry et al. (1998) comprueban la eficacia de la "viagra", en pacientes con disfunción eréctil, en un periodo de tratamiento de 28 días.

En suma, dada la presencia de limitaciones metodológicas tan importantes como las que acabamos de presentar, todo parece indicar un estado precientífico de las investigaciones sobre sexualidad y LM. Presentamos a continuación las principales conclusiones que pueden extraerse de esta revisión bibliográfica.

\section{CONCLUSIONES}

En nuestra revisión hemos realizado un recorrido por las publicaciones al uso en materia de LM y sexualidad. Esta panorámica de la bibliografia disponible la hemos centrado en el análisis del tipo de publicaciones sobre la sexualidad de las personas con LM, los grupos y áreas de interés estudiados y la metodología utilizada en las investigaciones. En función de lo obtenido tras la revisión de estas publicaciones podemos llegar a las siguientes conclusiones:

- Del volumen de publicaciones revisadas, 78 artículos, debemos destacar la gran cantidad de estudios de naturaleza teórica encontrados. En concreto, el 33,3\% de las publicaciones revisadas presentan estas características, versando sus contenidos sobre revisiones bibliográficas, reflexiones por parte de los autores y guías de orientación sexual. Estudios meramente descriptivos, reflexiones sobre lo ya descrito, que no aportan innovación o conocimiento sólidos sobre los que asentar la investigación futura.

- En cuanto a los trabajos de corte empírico, los estudio descriptivocorrelacionales $(61,54 \%)$ ocupan el abanico más amplio de publicaciones sobre la sexualidad de las PcLM. Por último, y con un nivel de representación mucho menor, encontramos los trabajos de corte experimental, es decir, aquellos en 
La sexualidad de las personas con lesión medular: aspectos psicológicos y sociales. Una revisión actualizada

los que los sujetos participan en algún tipo de terapia o tratamiento de rehabilitación sexual, y que suponen el 5,12\% del total de artículos revisados.

- En la mayor parte de los trabajos presentados se trabaja con muestras disponibles, es decir, se estudia aquello de lo que se dispone y se adapta la instrumentación a la muestra, dando la sensación, en muchas ocasiones, de algo forzado.

- Tienden a mezclarse los grupos sin hacer distinciones de sexo, edad, estado civil, status social u otras variables necesarias y fundamentales a la hora de realizar una investigación con el adecuado rigor científico.

- Inexistencia de cuestionarios o instrumentos estandarizados para evaluar el área de la sexualidad en los sujetos con LM. En la mayoría de los artículos no se especifican las pruebas que se pasan a los participantes.

- Predominio de carácter médico y neurofisiológico que queda patente en algunos de los trabajos. Quizá esto sea debido a que muchas de las investigaciones han sido llevadas a cabo en centros hospitalarios, y dirigidas principalmente por profesionales de la medicina.

- Práctica inexistencia de grupos control con los que poder comparar y contrastar los resultados encontrados en el grupo experimental.

- En aquellos artículos donde principalmente se estudian las relaciones de pareja y los cambios sufridos en sus relaciones sexuales después de sobrevenida la LM, las variables psicológicas (autoconcepto, nivel de ansiedad, superación personal, etc.), las sociales (familia, amigos, entorno, nivel de comunicación con la pareja, etc.) y las fisicas (nivel o grado de la lesión, movilidad, espasticidad, etc.), aparecen como elementos determinantes de cara a la rehabilitación y ajuste de la persona con LM.

- Se enfatiza el importante papel que juegan los profesionales en la rehabilitación sexual de este colectivo de PcLM. Sin embargo, son muchos los que admiten su desconocimiento sobre el tema, la falta de información al respecto y el no considerar el asesoramiento a las PcLM como parte fundamental o importante en su trabajo.

- La mayor parte de las publicaciones abogan por el "asesoramiento terapéutico" y la transmisión de una adecuada "información y educación sexual" como objetivo básico de la rehabilitación sexual de la persona con LM.

La investigación sobre este tema a lo largo de estas tres últimas décadas ha evolucionado muy poco, pese a constituir un aspecto relevante y de vital importancia en el proceso de adaptación y ajuste a la LM. En función de todo lo dicho y expuesto hasta el momento, creemos que el tema resulta lo suficientemente relevante como para continuar investigando sobre el mismo dado su estado precientífico y sus limitaciones metodológicas. Todo esto, sin perder de vista que una información más exhaustiva sería de gran utilidad para todo el equipo rehabilitador y, en último término, redundaría en una mejor y más adecuada adaptación de los programas de rehabilitación a las necesidades de las PcLM en el ámbito de la sexualidad. 


\section{BIBLIOGRAFÍA}

Aguado, A.L. (1995). Historia de las deficiencias. Madrid: Fundación ONCE.

Aguado, A.L. y Alcedo, M.A. (1995). Las personas con discapacidad física. En M.A. Verdugo (Dir), Personas con discapacidad: Perspectivas psicopedagógicas y rehabilitadoras (pp. 145-184). Madrid: Siglo XXI de España Editores.

Aguado, A.L. y Alcedo, M.A. (1999). Evaluación psicológica en rehabilitación. En A. Ruano, J.M. Muñoz y C. Cid (Coors), Psicología de la rehabilitación (pp. 33-58). Madrid: Editorial MAPFRE.

Aguado, A.L., Alcedo, M.A. y Rueda, B. (2003). ¿Investigación en discapacidad y envejecimiento?: Panorámica de las publicaciones sobre el envejecimiento de las personas con discapacidad. Análisis y modificación de conducta, 29 (125), 423-455.

Aguado, A.L., González, I., Alcedo, M.A. y Arias, B. (2003). Calidad de vida y LM. En M.A. Verdugo y B. Jordán de Urries (Coors), Investigación, innovación y cambio, (pp. 624-664). Salamanca: Amarú Ediciones.

Aguado, A.L., Rueda, B., Alcedo, M.A. y Arias, B. (2003). Evaluación del afrontamiento en personas con LM. En M.A. Verdugo y B. Jordán de Urries (Coors), Investigación, innovación y cambio, (pp. 665-686). Salamanca: Amarú Ediciones.

Alexander, C.J. (1991). Psychological assessment and treatment of sexual dysfunctions following spinal cord injury. The Journal of the American Paraplegia Society, 14 (3), 127-131

Anderson, C.J., Mulcahey, M.J. y Vogel, L.C. (1997). Menstruation and pediatric spinal cord injury. The Journal of Spinal Cord Medicine, 20 (1), 56-59.

Anderson T.P. y Cole T.M. (1975). Sexual counseling of the physically disabled. Postgraduate Medicine, 58 (1), 117-125.

Asociación Mundial de Sexología (1999). Declaración de los Derechos Sexuales. 14ํㅡㄹ Congreso Mundial de Sexología, Asamblea General de la Asociación Mundial de Sexología. Hong Kong, República Popular China. (Manuscrito xerocopiado).

Béjar y Béjar, D. (1989). Aspectos psíquicos de la sexualidad. En D. Béjar y Béjar (Coor), Paraplejia y Sexualidad (pp. 101-121).Valencia: ASPAYM.
Benony, H., Daloz, L., Bungener, C., Chahraoui, K., Frenay, C. y Auvin, J. (2002). Emotional factors and subjective quality of life in subjects with spinal cord injuries The American Journal of Physical Medicine and Rehabilitation, 81 (6), 437-445.

Berard, E.J. (1989). The sexuality of spinal cord injured women: physiology and pathophysiology. A review. Paraplegia, 27 (2), 99-112.

Berkam, A.H., Weissman, R. y Frielich, M.H. (1978). Sexual adjustment of spinal cord injured veterans living in the community. Archives of Physical Medicine and Rehabilitation, 59 (1), 29-33.

Black, K., Sipski, M.L. y Strauss, S.S. (1998). Sexual satisfaction and sexual drive in spinal cord injured women. The Journal of Spinal Cord Medicine, 21 (3), 240-244.

Blanchard, M. (1988). Educación sexual dirigida a los pacientes con lesiones de médula espinal y a sus enfermeros. En D. Krueger (Ed.), Psicología de la Rehabilitación (pp. 311-316). Barcelona: Herder.

Booth, S., Kendall, M., Fronek, P., Miller, D. y Geraghty, T. (2003). Training the interdisciplinary team in sexuality rehabilitation following spinal cord injury: A need assessment. Sexuality and Disability, 21 (4), 249-261.

Brucker, B.S. (1983). Spinal cord injuries. En T.G. Burish y L.A. Bradley (Comps.), Coping with Choronic Disease (pp. 285-311). Nueva York: Academic Press.

Burish, T.G. y Bradley, L.A. (1983). Coping with chronic disease: definitiones and issues. En T.G. Burish y L.A. Bradley (Eds), Coping with Chronic Disease (pp. 3-11). Nueva York: Academic Press.

Campo, M. y Sánchez, A. (2003). La mujer con LM: sexualidad y maternidad. Terapia Sexual y de Pareja, 16,4-18.

Camprubi, M. y Curcoll, M.L. (1976). La orientación del lesionado medular en el programa de rehabilitación. En Fundación Mapfre (Ed.), Orientación y formación profesional del minusuálido fisico (pp. 287-295). Madrid: MAPFRE.

Cardenal, F. (1993). Sexualidad y paternidad en personas con LM. Boletín del Real Patronato, 26, 82-93. 
Charlifue, S.W., Gerhart, K.A., Menter, R.R., Whiteneck, G.G. y Manley, M.S. (1992). Sexual issues of women with spinal cord injuries. Paraplegia, 30 (3), 192-199.

Chicano, L.A. (1989). Humanistic aspects of sexuality as related to spinal cord injury. The Jour nal of Neuroscience Nursing: Journal of the American Association of Neuroscience Nurses, 21 (6), 366369

Chigier, E. (1981). Sexuality and disability: the international perspective. En D.G. Bullard y S.E. Knight (Eds), Sexuality and Physical Disability: Personal Perspectives. St. Louis: C.V. Mosby.

Closson, J.B., Toerge, J.E., Ragnarsson, K.T., Parsons, K.C. y Lammertse, D.P. (1991). Rehabilitation in spinal cord disorders. Comprehensive managment of spinal cord injury. Archives of Physical Medicine and Rehabilitation, 72 (4), 298-308.

Cole, T.M. y Stevens, M.R. (1975). Rehabilitation professionals and sexual counseling for spinal cord injured. Archives of Sexual Behavior, 4 (6), 631-638.

Conine, T. (1988). Rehabilitación sexual: El papel del personal clínico. En D. Krueger (Ed.), Psicología de la Rehabilitación (pp. 107-114). Barcelona: Herder

Conine, T.A., Disher, C.S., Gilmore, S.L. y Fischer, B.A. (1979). Physical therapists knowledge of sexuality of adults with spinal cord injury. Physical Therapy, 59 (4), 395-398.

Courtois, F.J., Mathieu, C., Charvier, K.F., Leduc, B. y Belanger, M. (2001). Sexual rehabilitation for men with spinal cord injury: preliminary report on a behavioral strategy. Sexuality and Disability, 19 (2), 149-159.

Curcoll, M.L. y J. Vidal (Dirs) (1992). Sexualidad y LM. Barcelona: Fundación Instituto Guttmann

De Pinto, A. y Sánchez, A. (2000). LM: sexualidad y fertilidad. Madrid: Global Pharma Press.

Derry, F.A., Dinsmore, W.W., Fraser, M., Gardner, B.P., Glass, C.A., Maytom, M.C. y Smith, M.D (1998). Efficacy and safety of oral sildenafil (viagra) in men with erectile dysfunction caused by spinal cord injury. Neurology , 51 (6), 1629-1633.

Derry, F.A., Hultling, C., Seftel, A.D. y Sipski, M.L. (2002). Efficacy and safety of sildenafil citrate
(Viagra) in men with erectile dysfunction and spinal cord injury: a review. Urology, 60 (2), 49-57.

Donohue, J. y Gebhard, P. (1995). The Kinsey Institute/Indiana University report on sexuality and spinal cord injury. Sexuality and Disability, 13 (1), 7-85.

Drench, M.E. (1992). Impact of altered sexuality and sexual funtion in spinal cord injury: a review. Sexual and Disability, 10 (1), 3-14.

Ducharme, S.H. y Gill, K.M. (1997). Sexuality after spinal cord injury: answers to your questions. Boston.

Edmonson, B. (1988). Disability and sexual adjustment. En V.B. Van Hasselt, P.S. Strain y M. Hersen (Eds), Handbook of Developmental and Physical Disabilities (pp. 91-106). New York: Pergamon Press.

Evans, R.L., Halar, E.M., DeFreece, A.B. y Larsen, G.L. (1976). Multidisciplinary approach to sex education of spinal cord-injured patients. Physical Therapy, 56 (5), 541-545.

Ferolla, E.C. y Lourenco, C. (1996). Sexual orientation guide for patients with spinal cord injuries. Applied methodology and results. Revista Brasileira de Enfermagem, 49 (2),165-182.

Ferreiro-Velasco, M.E., Barca-Buyo, A., Salvador de la Barrera, S., Montoto-Marqués,A., Miguéns, X. y Rodríguez-Sotillo, A. (2004). Sexual issues in a sample of women with spinal cord injury. Spinal Cord, 43 (1), 51-55.

Fisher, T.L., Laud, P.W., Byfield, M.G., Brown T.T., Hayat, M.J. y Fiedler, I.G. (2002). Sexual health after spinal cord injury: a longitudinal study. Archives of Physical Medicine and Rehabilitation, 83 (8), 1043-1051.

Fitting, M.D., Salisbury, S., Davies, N.H. y Mayclin, D.K. (1978). Self-concept and sexuality of spinal cord injured women. Archives of Sexual Behavior, 7 (2), 143-156.

Fora i Eroles, F. (1992). Prólogo. En M.L. Curcoll y J. Vidal (Dirs), Sexualidad y LM (pp. 3-5). Barcelona: Fundación Instituto Guttmann.

Forner, J.V. (2001). Pasado, presente y futuro de la LM. En M. Giner, M. Delgado, I. Miguel, J.V. Forner y R. Miró (Dirs), LM: Guía de Autocuidados (pp.15-25). Valencia: Generalitat Valenciana, Conselleria de Sanitat. 
Freed, M.M. (1993). Lesiones traumáticas y congénitas de la médula espinal. En K. Lehman (Dir.), Krusen-Medicina Física y Rehabilitación (pp. 747-779). Buenos Aires: Editorial Médica Panamericana ( Orig. 1985).

Garcés, C. y Civera, A. (1997). Lesión en la médula espinal. Rol de Enfermería, 230, 23-30.

Giner, P.M. (2001). Alteraciones de la sexualidad y fertilidad. En M. Giner, M. Delgado, I Miguel, J.V. Forner y R. Miró (Dirs), LM: Guía de Autocuidados (pp. 118-129). Valencia: Generalitat Valenciana, Conselleria de Sanitat.

Girts, C. (1990). Nursing attitudes about sexuality needs of spinal cord injury patients. Rehabilitation Nursing: The Official Journal of the Association of Rehabilitation Nurses, 15 (4), 205-206.

Guldin, A. (2000). Self-claiming sexuality: mobility impaired people and american culture. Sexua lity and Disability, 18 (4), 233-238.

Halstead, L.S., Salhoot, J.T., Stock, D.D. y Sparks, R.W. (1978). Sexual attitudes, behavior and satisfaction for able-bodied and disabled participants attending workshops in human sexuality. Archives of Physical Medicine and Rehabilitation, 59 (11), 497-501.

Harrison, J., Glass, C.A., Owens, R.G. y Soni, B.M. (1995). Factors associated with sexual functioning in women following spinal cord injury. Paraplegia, 33 (12), 687-692.

Held, J.P., Cole, T.M., Held, C.A., Anderson, C. y Chilgren, R.A. (1975). Sexual attitude reassessment workshops: effect on spinal cord injured adults, their partners and rehabilitation professionals. Archives of Physical Medicine and Rehabilitation, 56 (1), 14-18.

Herson, L, Hart, K.A., Gordon, M.J. y Rintala, D.H. (1999). Identifying and overcoming barriers to providing sexuality information in the clinical setting. Rehabilitation Nursing: The Official Journal of the Association of Rehabilitation Nurses, 24 (4), 148-151.

Higgins, G.E. (1979). Sexual response in spinal cord injured adults: a review of the literature. Archives of Sexual Behavior, 8 (2), 173-196.

Ide, M. y Ogata, H. (1995). Sexual activities and concerns in persons with spinal cord injuries. Paraplegia, 33 (6), 334-337.
Katz, G. (1985). La vida sexual de los deficientes mentales. Bon Gen, 21, 27-54.

Kendall, M., Booth, S., Fronek, P., Miller, D. y Geraghty, T. (2003). The development of a scale to assess the training needs of professionals in providing sexuality rehabilitation following spinal cord injury. Sexuality and Disability, 21 (1), 49-64.

Kettl, P.(1991). Female sexuality after spinal cord injury. Sexual and Disability, 9 (4), 287-295.

Kreuter, M., Sullivan, M. y Siosteen, A. (1994a). Sexual adjustment after spinal cord injury (SCI) focusing on partner experiences. Paraplegia, 32 (4), 225-235.

Kreuter, M., Sullivan, M. y Siosteen, A. (1994b). Sexual adjustment after spinal cord injury-comparison of partner experiences in pre-and postinjury relationships. Paraplegia, 32 (11), 759-770.

Kreuter, M., Sullivan, M. y Siosteen, A. (1996). Sexual adjustment and quality of relationship in spinal paraplegia: a controlled study. Archives of Physical Medicine and Rehabilitation, 77 (6), 541548.

Lemon, M.A. (1993). Sexual counseling and spinal cord injury. Sexual and Disability, 11 (1), 7397.

Leibowitz, R. (2003). Sexual challenges and creative solutions: voices of women with spinal cord injury. Dissertation Abstracts International: Section B: The Sciences \& Engineering, 64 (2-B), 967.

Lesky, J. (2001). Needs of paraplegics with respect to psychological care during initial rehabilitation. A retrospective survey. Rehabilitation, 40 (2), 76-83.

López, F. (1993). Criterios de salud sexual y minusvalias. En Instituto Nacional de Servicios Sociales (Ed.), Sexualidad en personas con minusvalía psíquica (ps 21-40). Madrid: IMSERSO.

Low, W.Y. y Zubir, T.N. (2000). Sexual issues of the disabled: implications for public health education. Asia-Pacific Journal of Public Health, 12 (Suplemento), 78 - 83.

Lysberg, K. y Severinsson, E. (2003). Spinal cord injured women's views of sexuality: a norwegian survey. Rehabilitation Nursing: The Official Journal of the Association of Rehabilitation Nurses, 28 (1), 23-26. 
La sexualidad de las personas con lesión medular: aspectos psicológicos y sociales. Una revisión actualizada

McAlonan, S. (1996). Improving sexual rehabilitation services: the patient's perspective. The American Journal of Occupational Therapy: Official Publication of the American Occupational Therapy Association, 50 (10), 826-834

McBride, K.E. y Rines, B. (2000). Sexuality and spinal cord injury: a road map for nurses. SCI Nursing: a publication of the American Association of Spinal Cord Injury Nurses, 17 (1), 8-13.

Miguel, I. (2001). Epidemiologia de la LM. En M. Giner, M. Delgado, I. Miguel, J.V. Forner y R. Miró, LM: Guía de Autocuidados (pp. 47-59). Valencia: Generalitat Valenciana, Conselleria de Sanitat.

Miller, D.K. (1975). Sexual counseling with spinal cord-injured clients. Journal of Sex \& Marital Therapy, 1 (4), 312-318.

Milligan, M.S. y Neufeldt, A.H. (2001). The mit. of asexuality: a survey of social and empirical. Sexuality and Disability, 19 (2), 91-110.

Novak, P.P. y Mitchell, M.M. (1988). Professional involvement in sexuality counseling for patients with spinal cord injuries. The American Journal of Occupational Therapy: Official Publication of the American Occupational Therapy Association, 42 (2), 105-112.

OMS (1974). Serie de Informes Técnicos, 572, 6.

Pentland, W., Walker, J., Minnes, P., Tremblay, M., Brouwer, B. y Gould, M. (2002). Women with Sspinal cord injury and the impact of aging. Spinal Cord, 40 (8), 374-387.

Pervin-Dixon, L. (1988). Sexuality and the spinal cord injured. Journal of Psychosocial Nursing \& Mental Health Services, 26 (4), 31-34.

Phelps, G., Brown, M., Chen, J., Dunn, M. Lloyd, E., Stefanick, M.L., Davidson, J.M. y Perkash, I. (1983). Sexual experience and plasma testosterone levels in male veterans after spinal cord injury. Archives of Physical Medicine and Rehabilitation, 64 (2), 47-52.

Phelps, J., Albo, M., Dunn, K. y Joseph, A. (2001). Spinal cord injury and sexuality in married or partnered men: activities, function, needs, and predictors of sexual adjustment. Archives of Sexual Behavior, 30 (6), 591-602.

Reitz, A., Tobe, V., Knapp, P. y Schurch, B (2004). Impact of spinal cord injury on sexual health and quality of life. International Journal of Impotence Research, 16, 167-174.

Richards, E., Tepper, M., Whipple, B. y Komisaruk, B.R. (1997). Women with complete spinal cord injury: a phenomenological study of sexuality and relationship experiences. Sexuality and Disability, 15 (4), 271-283.

Rohme, M. (1979). The public health nurse as sexual counselor for spinal cord injured men. Sexuality and Disability, 2 (1), 8-15.

Sawyer, H.W. y Allen, H.A. (1983). Sexuality and spinal cord injured individuals: a challenge for counselors and trainers. Journal of Applied Rehabilitation Counseling, 14 (4), 14-17.

Sawyer, H.W., Allen, H.A. y Reisin, B.L. (1983). Microcounseling: a counselor training format relating to sexual concerns of spinal cord injured women. Sexuality and Disability, 6 (1), 30-37.

Singer, L.R. (2003). Adjustment to spinal cord injury: a critical review. Dissertation Abstracts International: Section B: The Sciences \& Engineering, 63 (10-B), 4925.

Siosteen, A., Lundqvist, C., Blomstrand, C., Sullivan, L. y Sullivan, M. (1990). Sexual ability, activity, attitudes and satisfaction as part of adjustment in spinal cord-injured subjects. Paraplegia, 28 (5), 285-295.

Sipski, M.L. (1993). The impact of spinal cord trauma on female sexual function. En F. Haseltine y S. Cole (Eds), Reproductive Issues for Persons with Physical Disabilities (pp.209-220). Virginia.

Sipski, M.L. (1998). Sexual functioning in the spinal cord injured. International Journal of the Impotence Research: Official Journal of the International Society for Impotence Research, 10 (Suplemento), 128-130.

Sipski, M.L. y Alexander, C.J. (1993). Sexual activities, response and satisfaction in women preand post-spinal cord injury. Archives of Physical Medicine and Rehabilitation, 74 (10), 1025-1029.

Sjogren, K. y Egberd, K. (1983). The sexual experience in younger males with complete spinal cord injury. Scandinavian Journal of Rehabilitation Medicine Supplement, 9, 189-194.

Spica, M.M. (1989). Sexual counseling standards for the spinal cord-injured. The Journal of 
Neuroscience Nursing: Journal of the American Association of Neuroscience Nurses, 21 (1), 56-60.

Teal, J.C. y Athelstan, G.T. (1975). Sexuality and spinal cord injury: some psychosocial considerations. Archives of Physical Medicine and Rehabilitation, 56 (6), 264-268.

Taleporos, G. y McCabe, M.P. (2001). Physical disability and sexual steem. Sexuality and Disability, 19 (2), 131-148.

Tepper, M.S. (1997). Living with a disability: a man's perspective. En M. Sipski y C. Alexander (Eds), Sexual Function in People with Disability and Chronic Illness: A Health Professional's Guide (pp.133-146). Huntington.

Tepper, M.S. (2002). Lived experiences that impede or facilitate sexual pleasure and orgams in people with spinal cord injury. Dissertation Abs tracts International Section A: Humanities \& Social Sciences, 62 (11-A), 3706.

Tepper, M.S., Whipple, B., Richards, E. y Komisaruk, B.R. (2001). Women with complete spinal cord injury: a phenomenological study of sexual experiences. Journal Sex \& Marital Therapy, 27 (5), 615-623.

Ulmer, D.B. (1990). Special needs of the young spinal cord injured patient in a nursing home. SCI Nursing: a Publication of the American Association of Spinal Cord Injury Nurses, 7 (2), 27-30.

Wada, M. y Brodwin, M. (1975). Attitudes of society toward sexual functioning of male individuals with spinal cord injury. Psychology: A Journal of Human Behavior, 12 (4), 18-22.

Ward-McKinlay, T., Botvinn-Madorsky, J.G. y Ward-McKinlay, C. (1983). Sexuality and disability. Family Therapy Collections, 5, 129-152.

Westgren, N., Hultling, C., Levi, R., Seiger, A. y Westgren, M. (1997). Sexuality in women with traumatic spinal cord injury. Acta Obstetricia et Ginecologica Scandinavica, 76 (10), 977-983.
Whipple, B., Richards, E., Tepper, M. y Komisaruk, B.R. (1996). Sexual response in women with complete spinal cord injury. Sexuality and Disability, 14 (3), 191-201.

White, M.J., Rintala, D.H., Hart, K.A., Young, M.E. y Fuhrer, M.J. (1992). Sexual activities, concerns and interests of men with spinal cord injury. Archives of Physical Medicine and Rehabilitation, 71 (4), 225-231.

Willmuth, M.E. (1987). Sexuality after spinal cord injury: a critical review. Clinical Psychology Review, 7 (4), 789-412.

Yoshida, K.K. (1994). Intimate and marital relationships: an insider's perspective. Sexuality and Disability, 12 (3), 179-189.

Zaretsky, H.H. (1981). Sexualidad e incapacidad fisica. En H.H. Zaretsky y L.P. Ince (Eds), Terapia de Conducta en Rehabilitación (pp. 165-236). Madrid: Fundación MAPFRE.

Zwerner, J. (1982). A study of issues in sexuality counseling for women with spinal cord injuries. Women \& Therapy, 1 (3), 91-100.

\section{WEBGRAFÍA}

Hospital Nacional de Parapléjicos: www.infomedula.org.

Instituto de Neurorrehabilitación Guttmann: www.guttmann.com.

Fundación Christopher Revé: www.apacure. com.

Proyecto Miami para la cura de la parálisis: www.miamiproject.miami.edu.

Proyecto Stand Up and Walk: www.comunidadescolar.pntic.mec.es, www.ctv.es y www.profesional.medicinetv.com. 